\title{
COVID-19 in Children and Adolescents with Endocrine Conditions
}

Authors

Eirini Kostopoulou1 * (D), Maria Güemes ${ }^{2}$, Pratik Shah ${ }^{3}$ (D)

Affiliations

1 Division of Paediatric Endocrinology and Diabetes, University of Patras, Patras, Greece

2 Endocrinology Service, Hospital Infantil Universitario Niño Jesús, Madrid, Spain

3 Paediatric Endocrinology and Diabetes, The Royal London Children's Hospital, London, United Kingdom

Key words

COVID-19, pediatrics, endocrine disease, diabetes mellitus, psychological aspects

received 14.05 .2020

accepted after revision $\quad 20.07 .2020$

published online $\quad 09.09 .2020$

Bibliography

Horm Metab Res 2020; 52: 769-774

DOI 10.1055/a-1227-6635

ISSN 0018-5043

(C) 2020. Thieme. All rights reserved.

Georg Thieme Verlag KG, Rüdigerstraße 14, 70469 Stuttgart, Germany

\author{
Correspondence \\ Dr. Pratik Shah \\ Consultant in Paediatric Endocrinology and Honorary \\ Clinical Lecturer, The Royal London Children's Hospital \\ Barts Health NHS Trust \\ E1 1FR London \\ United Kingdom \\ Tel.: + 447704639358 \\ pratik.shah6@nhs.net,drshahp@gmail.com
}

\section{ABSTRACT}

The rapid spread of coronavirus disease (COVID-19) worldwide justifies global effort to combat the disease but also the need to review effective preventive strategies and medical management for potentially high-risk populations during the pandemic. Data regarding the COVID-19 manifestations in adults with underlying endocrine conditions, especially diabetes mellitus, are increasingly emerging. Albeit children and adolescents are considered to be affected in a milder manner, paucity of information regarding COVID-19 in children who suffer from endocrinopathies is available. The present review comprehensively collects recommendations issued by various health organizations and endocrine associations for the management of pediatric endocrine conditions during the pandemic. Adhering to the specific "sick day management rules" and undelayed seeking for medical advice are only needed in most of the cases, as the vast majority of children with endocrine disorders do not represent a high-risk population for contamination or severe presentation of COVID-19. Psychological implications in these children and adolescents are also considered.

\section{Introduction}

On December $31^{\text {st }}$ 2019, the World Health Organization (WHO) were notified about a cluster of pneumonia cases of unknown cause detected in the city of Wuhan in Hubei province, China [1]. Investigations showed that the causing factor was a previously unknown virus, the severe acute respiratory syndrome-coronavirus-2 (SARS-CoV-2) and the relative disease was named coronavirus disease 2019 (COVID-19) [1] and was characterized as a pandemic on March $11^{\text {th }}$ 2020 [1]. According to a recent update by WHO on April 19th 2020 , laboratory confirmed cases for COVID-19 have risen to 2245872 worldwide, including 152707 deaths [2]. Reasonably, in the case of

\footnotetext{
These authors contributed equally to this manuscript
}

such a massive health crisis as the COVID-19 outbreak, questions regarding increased vulnerability in specific population groups arise. One of the potentially high-risk populations is the pediatric population and particularly children with chronic diseases.

It has become evident that the disease can occur amongst all age groups, including the pediatric population [3]. However, early data suggest that the impact of the COVID-19 pandemic in children is modest [4], as it presents less frequently and with milder symptoms and severity [3]. In a population-based study in Iceland, children under 10 years of age and females had a lower incidence of SARS-CoV-2 infection than adolescents or adults and males [5]. The rate of mortality is reported to be much lower in 
children $(<0.1 \%$ ) compared to adults [6]. Suggested possible mechanisms for the milder clinical picture in children include: i) age-related high nasal gene expression of the angiotensin-converting enzyme 2 (ACE2) receptor (the functional receptor of SARSCov-2) in children aged $>10$ years [7]; ii) a constitutional high lymphocyte count; and iii) trained immunity, an innate immune memory formed by "memory cells" after antigen exposure [8, 9].

Thus far, limited data are available about the possible consequences of COVID-19 on children and adolescents with a chronic illness. The Royal College of Paediatrics and Child Health (RCPCH) in the UK have published "shielding" guidance for children and young people with recent transplantation, immunosuppression, hemato-oncological, cardio-respiratory, renal, and gastrointestinal disorders [10]. Also, the British Society of Gastroenterology has published recommendations for management of inflammatory bowel disease during the COVID-19 pandemic [11]. However, to our knowledge, no reliable data exist associating endocrine disorders and vulnerability to COVID-19, expressed as increased morbidity or mortality and development of endocrine complications related to SARS-Cov-2.

\section{Materials and Methods}

The present review summarizes the current knowledge on the possible impact of COVID-19 in children and adolescents with endocrine disorders, considering known and newly developed recommendations from Health and Endocrine societies. Pubmed search for publications was performed in English, Spanish and Greek as of April $18^{\text {th }}, 2020$. The search terms that were used included "COVID19”, “children”, “endocrine diseases”, “diabetes”, "thyroid diseases”, “hypopituitarism”, “hypoadrenalism”, “hypoglycemia”, “Cushing syndrome", "obesity”. The following literature review and recommendations should be read in light of limitations, such as the rapidly evolving knowledge and recently gained experience regarding COVID-19, which also predominantly involves adults.

\section{Discussion}

To date, none of the endocrine conditions have been classified as predisposing factors for the Covid-19 infection [12] and hence, children with endocrine diseases have not shown a different disease pattern compared to children without an endocrine disorder. Nonetheless, there are multifaceted endocrine implications of COVID-19.

As for the general population, since SARS-Cov-2 is a virus of particularly increased emergence and spread capacity, children and adolescents with endocrine disorders are strongly encouraged to adhere to preventive and protective measures against viral spread, such as social distancing, home confinement, self-hygiene (hand-washing, avoidance of touching their face, coughing/sneezing into elbow or tissue) and disinfecting frequently-touched surfaces [13]. Furthermore, adherence to local regulations regarding general preventive measures and schooling is of major importance [13]. Remote learning from home, if plausible, is the safest method [12].

In the case of symptoms of infection, such as fever, cough, and dyspnoea in particular, medical advice should be sought and if the symptoms are of increasing severity, visiting the nearest hospital and taking all the necessary measures (e. g., face mask), is imperative. Like with any other pediatric infection, it is important to maintain adequate hydration with frequent fluid intake. If COVID-19 is confirmed, recommended control measures should be promptly implemented together with supportive management of complications [14]. If hospitalization is needed, the health care team should be aware so as to modulate management, particularly in endocrine diseases such as type 1 diabetes mellitus, hypoglycemia disorders and adrenal insufficiency.

Furthermore, it is important that children with endocrine diseases who receive replacement or supplementation therapy maintain a euhormonal status. The necessity of dose adjustment according to the individual needs should be discussed with the treating physician, since no universal recommendation is applicable in all cases. Also, sufficient supply of medications should be ensured at reasonable quantities [12]. Routine hospital visits should be avoided for nonurgent reasons so that exposure to COVID-19 is restricted and those should be replaced by telephone or video consultation to maintain an optimal control of the underlying disease $[15,16]$. This requires robust telephone triage and expansion of telehealth visits $[16,17]$.

- Table 1 collects pivotal aspects for managing children and adolescents with endocrine disorders during the COVID-19 pandemic.

\section{Endocrine Diseases During COVID-19 - Recommendations}

\section{Diabetes Mellitus (DM)}

In adults, reports from China [18], Italy [19], and USA [20] indicate that DM is a risk factor for severe COVID-19 disease. Longstanding DM involves low-grade chronic inflammation, which may promote the cytokine storm that seems to be implicated in the severe evolution of COVID-19, as inflammation markers (C-reactive protein, fibrinogen, D-dimer, ferritin, erythrocyte sedimentation rate, IL-6)

- Table 1 Key principles for managing children and adolescents with endocrine disorders during the COVID-19 pandemic.

- Promote adherence to protective infection control measures (hand hygiene, face mask, social distancing, etc.).

- Provision of reliable information by the health care team so that anxiety related to misinformation is prevented.

- Reassurance of the parents and children/adolescents that the vast majority of children with endocrine disorders do not represent a high-risk population for contamination or severe presentation of COVID-19.

- In case of COVID-19 infection, following the specific "sick day management rules" and seeking for medical advice without delay are adequate in most cases for an optimal outcome.

- Parents should also be reinforced by clinicians to be involved in entertaining children's anxiety, ensuring good nutritional/fluid intake, active monitoring, and offering supportive therapy for their children. 
have been found higher in patients with DM [21]. Hyperglycemia due to the COVID-19-induced stress, hypoglycemia or sharp glycemic excursions entail detrimental outcomes for DM patients [21]. The identified data from adult studies do not specifically differentiate type 1 from type 2 DM.

With regard to children and adolescents with DM, it is expected that those with type $1 \mathrm{DM}$ and good metabolic control will follow the same course of illness as their peers $[12,13]$. Nonetheless, those with a poor control could have a debilitated immunity that puts them at a greater risk for contamination from SARS-Cov-2 [12], and a more severe presentation [22].

The European Society for Paediatric Endocrinology (ESPE) recommends maintaining the usual amount of back-up insulin supplies, for at least a week in advance, without stockpiling copious quantities, as this could jeopardize the supply chain leading to territorial or worldwide scarcity [12].

In the instance of symptoms that could be related to COVID-19, such as fever, cough or shortness of breath, it is paramount that medical assistance is sought without delay [13]. As during any other intercurrent illness, glycemic control may deteriorate, hence following "sick day rules" also apply in the case of COVID-19 [13], as well as contacting the therapeutic team $[12,16]$. Recommendations from the International Society for Pediatric and Adolescent Diabetes (ISPAD) include [13]: i) to monitor blood glucose and ketone bodies more frequently; ii) to target blood glucose concentrations between 70-180 mg/dl (4-10 mmol/I) and negative ketones during illness; iii) to never discontinue insulin administration, since increased insulin requirements are expected in case of fever; to watch and support hydration, especially in the presence of fever and/or vomiting/diarrhea; and iv) to treat the underlying sickness and symptoms. Should there be signs of metabolic decompensation (namely dehydration, hyperglycemia with persistent ketosis, hyperventilation, exhaustion), urgent assessment by health care providers is deemed necessary [13]. A pediatric case series from Italy reports delayed access to health care secondary to fear of COVID-19, including two cases of type 1 DM onset with severe ketoacidosis requiring intensive care unit (ICU) admission due to late access to the hospital [23].

\section{Adrenal Insufficiency (congenital and acquired primary adrenal insufficiency, secondary adrenal insufficiency, adrenal suppression after long-term steroid medication)}

To date, there is no evidence that patients with adrenal insufficiency are at increased risk of contracting COVID-19. Nonetheless, patients with primary adrenal insufficiency (e. g., congenital adrenal hyperplasia) are slightly more susceptible to infections in general. This may partly be explained by the impaired natural immunity function characterized by a defective action of neutrophils and natural killer cells, which is known to be associated with primary adrenal insufficiency [24]. Furthermore, susceptibility to infections may also be explained by an insufficient increase of the hydrocortisone dosage at the beginning of an infection. Therefore, recommendations suggest that, if asymptomatic, children should remain on regular replacement doses of hydrocortisone and not increased doses. If symptoms suggestive of COVID-19 develop (fever, cough, dyspnoea, vomiting, diarrhoea), the "sick day rules" are recommended, including immediately increasing the hydrocortisone doses (e.g., $>38^{\circ} \mathrm{C}$ : 2 -fold increase, $>39^{\circ} \mathrm{C}$ : 3 -fold increase) until the fever has subsided and adding an extra doubled dose [12]. Adequate hydration is also recommended, particularly in the presence of fever [12]. In the case of more severe symptoms, the health care team should be contacted for further advice and if the medication cannot be orally received due to vomiting, urgent medical care should undoubtedly be sought and parenteral glucocorticoids initiated in the form of intramuscular injection of hydrocortisone, accompanied by glucose, preferably in the form of oral gel [25].

Among patients with COVID-19, it is of major importance to recognize those with a history of possible adrenal suppression secondary to prior exposure to glucocorticoids for more than 3 months, so that parenteral treatment with glucocorticoids is considered [22]. Physiological stress doses of hydrocortisone and not pharmacological doses are recommended in this case.

Of note, it has been proposed that all the patients receiving corticosteroids for other medical reasons and not because of adrenal insufficiency or adrenal suppression, should be considered as highrisk patients for contracting COVID-19 and experiencing more severe symptoms [22]. Supraphysiologic doses of glucocorticoids may further increase susceptibility to COVID-19 due to their immunosuppressive effect.

\section{Hypopituitarism}

Children diagnosed with hypopituitarism are not at increased risk for COVID-19. As a significant percentage of these patients have secondary adrenal insufficiency, the same recommendations apply as for children with adrenal insufficiency [12]. Identical advice applies for each of the endocrine deficiencies involved.

Particular attention should be given to children with craniopharyngioma, the most common pediatric tumor in the hypothalamic and pituitary region and an important cause of hypopituitarism in children. In this case, the endocrine deficiencies are attributable to either the tumor itself, due to pressure on the pituitary and hypothalamus, or the operative procedure and/or irradiation following the diagnosis. Tumor location or surgical intervention are also the etiology of the hyperphagia observed in these patients [26], which results in hypothalamic obesity and increased morbidity and mortality rates due to the associated metabolic derangement. Due to the complicated pre- and post-operative course of the disease, the treating health care team should be aware of a possible infection with COVID-19, in order to guide treatment decisions.

\section{Diabetes Insipidus}

In addition, in the case of the presence of both secondary adrenal insufficiency and diabetes insipidus, it is important that medications for both conditions are always received. Also, careful monitoring of fluid intake and urinary losses is important, as well as judicious replacement of water, in order to avoid hypo- or hypernatremia, particularly in the presence of fever, tachypnea and the co-existence of impaired ability for fluid intake due to altered level of consciousness [27].

Since hydrocortisone is essential for the clearance of excess water through the kidneys, it should be administered at adequate doses to avoid accumulation of fluid, particularly if the child also receives des- 
mopressin. Therefore, the dose of hydrocortisone should be immediately doubled in the presence of a symptomatic COVID-19. In such patients, urination should be monitored closely. If urination is reduced or stopped, desmopressin should be discontinued. If the child has an intact thirst mechanism, fluid intake will be adequate. If the thirst mechanism is not intact, maintenance fluids should be administered, with additional fluids to replace urinary losses [27]. The therapeutic team should be aware of the patient's condition in this scenario.

\section{Thyroid Disorders (Hypothyroidism/ Hyperthyroidism)}

Children with thyroid disease, even if poorly controlled, are not at increased risk of COVID-19 and no extra measures are needed than those that apply to the general population [28]. However, patients with poorly controlled thyroid disease (e. g., thyrotoxicosis) may be at higher risk of complications from an infection [28]. Furthermore, it is well established that autoimmune thyroid disease does not cause immunosuppression [28]. Medications used for the treatment of thyroid disease, including thyroxine, carbimazole, methimazole, and propylthiouracil do not affect the immune function and do not pose the patients at a heightened risk for COVID-19 [28].

Recommendations from the British Thyroid Association about adults with thyroid eye disease who are on high-dose steroid or mycophenolate or rituximab and are considered as immunocompromized and susceptible to infections, include following the confinement and health protection measures very strictly [28]. Depending on the severity of the eye problem, high-dose steroids or immunosuppressives might need to be suspended [28]. No different recommendations exist for the pediatric population.

Furthermore, for patients who had radioiodine therapy or thyroid surgery for benign thyroid disease, no evidence is available to suggest that these patients are at increased risk of a viral infection [28].

With regard to the treatment when COVID-19 is confirmed in an adult or a pediatric patient, the normal dose of thyroxine should be continued. If gastrointestinal disturbances are severe, the dose may need to be repeated when feasible [28]. In the case of symptoms that may be related to COVID-19, patients with hyperthyroidism who are on anti-thyroid drugs should immediately suspend the medication and be tested for possible agranulocytosis by performing a full blood count, as the symptoms of agranulocytosis (sore throat, mouth ulceration, fever, flu-like illness) may overlap with those from COVID-19 [28].

\section{Hypoglycemia (hyperinsulinemic hypoglycemia and ketotic hypoglycemia)}

Receiving the proper medications and regular glucose monitoring should be ensured in children who are prone to have hypoglycemia during illness, especially those with ketotic hypoglycemia or hyperinsulinemic hypoglycemia. These children are not considered vulnerable, with the exception of a minority that receive Sirolimus, a mammalian target of rapamycin (mTOR) inhibitor with immunosuppressive action. For the latter, strict hygiene and confinement measures should be taken during the COVID-19 pandemic. Adequate hydration (small volumes of fluid at frequent intervals) is also highly recommended [29]. In addition, side effects of the medications used to treat hyperinsulinemic hypoglycemia (e. g., diazoxide: water retention and pulmonary hypertension; somatostatin analogues: cardiac arrhythmias and cardiac conduction disorders) should be taken into consideration in the case of COVID-19.

During this pandemic, children should follow the "sick-day rules" for hypoglycemia, which include close monitoring of glucose levels, adequate hydration, ensuring availability of medications and emergency regime, preferably in the form of a glucose gel/juice or glucose powder solution, and contacting the medical team in the instance of hypoglycemia of unusual frequency and severity.

\section{Cushing Syndrome}

Cushing syndrome represents an immunocompromised state, which can increase susceptibility to infections, hence COVID-19 also [30]. Susceptibility to opportunistic infections is caused by prolonged exposure to elevated cortisol concentrations. Glucocorticoids have a potent anti-inflammatory and immunosuppressive action, resulting in the suppression of cellular and humoral immune functions. Hyperglycemia in patients with Cushing syndrome resulting from increased insulin resistance, further contributes to immunosuppression. The higher the concentrations of circulating cortisol, the more severe the infections. Therefore, adherence to preventive self-protective measures, complying with the regular treatment and seeking medical advice in the case of even minor symptoms, is crucial.

\section{Hypoparathyroidism}

Children with hypoparathyroidism have no susceptibility to infections, unless hypoparathyroidism exists in the context of 22q11.2 deletion syndrome, which in the majority (64-77\%) of the patients, encompasses immunodeficiency of variable severity. In the case of 22q11.2 deletion syndrome, the predominant cause of immunodeficiency is compromised T cell production due to thymic hypoplasia or aplasia, and recurrent infections of the respiratory tract, albeit usually not severe, they represent a common clinical manifestation. Therefore, susceptibility to COVID-19 is increased and special attention should be paid during COVID-19 infection.

If COVID-19 is confirmed and the child is unable to receive oral tablets, parenteral treatment should be initiated with intravenous calcium. Of note, hypocalcaemia may worsen breathing capacity due to weakening of the chest muscles [31].

\section{Obesity}

It is well established in the literature that excess weight promotes immune dysregulation and chronic inflammation, which result in the cytokine storm associated with the Acute Respiratory Distress Syndrome seen in influenza and other viral infections [32]. Limited data is available regarding the impact of COVID-19 in patients with obesity. It has been reported that severe obesity may be a risk factor for respiratory complications during the course of COVID-19 [12]. It has also been reported that young people suffering from severe obesity may develop destructive alveolitis resulting in respiratory failure and death (author's personal experience) [16]. In addition, it is well known that obesity is associated with sleep apnoea syndrome and surfactant dysfunction, which may worsen the clinical course of COVID-19 infection. Therefore, it is important that children with obesity, and those with severe obesity in particular, strictly follow self-protective strategies to avoid COVID-19. 
For these patients, COVID-19 may have an additional negative impact on obesity itself. Due to the confinement measures that include school closure and prohibition of outdoor exercise or exercise in a gymnasium, regular exercise may be restricted. Furthermore, home restriction can lead to increased caloric intake and especially of calorie-dense comfort foods. Additionally, sedentary activities and screen time are expected to expand [33]. For these reasons, it is estimated that the COVID-19 pandemic may result in exacerbation of childhood obesity [33]. It is important that these patients continue exercise through home-based exercise programs and that they follow a well-balanced diet.

\section{COVID-19 and Psychological Consequences in Children and Adolescents with Endocrine Disorders}

It becomes evident that as the COVID-19 pandemic peaks, it is causing widespread concern, fear and stress. This is understandable due to the high morbidity and mortality rates of the disease in adults, the restrictive preventive measures that result in a drastic change of everyday life, affecting work, education, social life, entertainment, and the long period sustained fear of the unknown outcome of this outbreak [34-40].

The impact of the COVID-19 pandemic to the pediatric population may be worsened by the co-existence of a chronic disease. It is well established that a chronic illness may impair the immunologic reaction of an individual to infections. Specifically, chronic stress from a permanent disease may erode immunologic mediators, increase risk of infections and cytokine production and decrease antibodies and defensive mechanisms [41-43].

On the other hand, children suffering from a chronic disease are already experiencing higher stress and additional pressure compared to healthy children due to the need for compliance to chronic medication and regular doctor visits, but also because of school disruption and fear of death [44-46]. Children's stress due to a chronic endocrine disease can be magnified by a pandemic, as children are likely to experience anxiety and fear of death or of their relatives dying and they may also see the supportive family structure previously provided, to fall apart. Of note, parental anxiety about children exposure to COVID-19, potentially further complicating their chronic endocrine disease, should not be ignored. This may lead to retaining children out of school, thereby adding to social marginalization of the patients as well as aggravating their feeling of distress and impacting their wellbeing.

Hence, therapeutic teams should be aware of the possible psychological consequences of the current pandemic and monitor psychological responses, even screen for psychological distress using psychometric tools, such as validated questionnaires. This could promote the implementation of stress management strategies and interventions in order to avoid potential future psychological distress that may affect the feeling of wellbeing and adherence to treatment, allowing an optimal control and outcome of the endocrine disease.

\section{Conclusions}

The COVID-19 pandemic represents the greatest global public health care crisis since the influenza outbreak in 1918. Thus far, the available data on the impact of COVID-19 on patients with endocrine disorders is reassuring for the majority of the cases. In case of COVID-19 infec- tion, "sick day" rules apply. However, lack of sufficient and reliable data, particularly for the pediatric population, should prompt health care providers to be more vigilant in the assessment of children with endocrine disorders and concerning symptoms, such as fever and dyspnea.

\section{Author Contributions}

PS contributed to the conception of the manuscript. EK and MG designed and drafted the article. EK, MG, and PS revised the content and gave final approval of the version.

\section{Acknowledgements}

In memoriam Alysha Keshani, former colleague and Endocrine dietician at Great Ormond Street Hospital for Children NHS Foundation Trust, London (UK).

\section{Conflict of Interest}

The authors declare that they have no conflict of interest.

\section{References}

[1] World Health Organization (WHO). Rolling updates on coronavirus disease (COVID-19). Internet, cited 18 April 2020; Available from: https://www.who.int/emergencies/diseases/novel-coronavirus-2019/ events-as-they-happen

[2] World Health Organization (WHO), Regional Office for Europe. COVID-19: Latest updates. Internet, cited 19 April 2020; Available from: http://www.euro.who.int/en/health-topics/health-emergencies/ coronavirus-covid-19/covid-19-latest-updates

[3] Dong Y, Mo X, Hu Y et al. Epidemiology of COVID-19 Among Children in China. Pediatrics 2020; 145 pii: e20200702, doi:10.1542/ peds.2020-0702 [Epub ahead of print]

[4] Livingston E, Bucher K. Coronavirus Disease 2019 (COVID-19) in Italy. JAMA 2020; 323: 1335

[5] Gudbjartsson DF, Helgason A, Jonsson $\mathrm{H}$ et al. Spread of SARS-CoV-2 in the Icelandic Population. N Engl J Med 2020; 382: 2302-2315

[6] Promislow DEL. A geroscience perspective on COVID-19 mortality. J Gerontol A Biol Sci Med Sci 2020, pii: glaa094 doi:10.1093/gerona/ glaa094 [Epub ahead of print]

[7] Bunyavanich S, Do A, Vicencio A. Nasal gene expression of angiotensin-converting enzyme 2 in children and adults. JAMA 2020; 323: 2427-2419

[8] Cristiani L, Mancino E, Matera L et al. Will children reveal their secret? The coronavirus dilemma. Eur Respir J 2020; 2000749. doi: 10.1183/13993003.00749-2020 [Epub ahead of print]

[9] Cao Q, Chen Y-C. SARS-CoV-2 infection in children: Transmission dynamics and clinical characteristics. J Formos Med Assoc 2020; 119: 670-673

[10] Royal College of Paediatrics and Child Health (RCPCH). COVID-19 'shielding' guidance for children and young people. Internet, cited 20 April 2020; Available from: https://www.rcpch.ac.uk/sites/default/ files/generated-pdf/document/COVID-19-\%2527shielding \%2527-guidance-for-children-and-young-people.pdf

[11] Kennedy NA, Jones G, Lamb CA et al. British Society of Gastroenterology guidance for management of inflammatory bowel disease during the COVID-19 pandemic. Gut 2020, doi:10.1136/gutjnl-2020-321244 [Epub ahead of print] 
[12] European Society for Paediatric Endocrinology (ESPE). Coronavirus disease (COVID-19) information for children and adolescents living with endocrine conditions, including type 1 diabetes mellitus. Internet, cited 23 Mar 2020; Available from: https://www.eurospe.org/ news/item/14064/COVID-19-information-for-children-and-adolescents-living-with-endocrine-conditions-including-type-1-diabetes-mellitus

[13] International Society for Pediatric and Adolescent Diabetes (ISPAD). Coronavirus infection (COVID-19). Summary of recommendations regarding COVID-19 in children with diabetes. Internet, cited $19 \mathrm{Mar}$ 2020; Available from: https://www.ispad.org/page/CoronavirusinfectionCOVID-19

[14] Centers for Disease Control and Prevention. Coronavirus disease 2019 (COVID-19) clinical care. Internet, cited 30 Mar 2020; Available from: https://www.cdc.gov/coronavirus/2019-ncov/hcp/clinical-guidance-management-patients

[15] International Diabetes Federation. COVID-19 outbreak: guidance for people with diabetes. Internet, cited 15 April 2020; Available from: idf. org/our-network/regions-members/europe/europe-news/196-information-on-corona-virus-disease-2019-covid-19-outbreak-and-guidance-for-people-with-diabetes.html

[16] Puig-domingo M, Marazuela M, Giustina A. COVID-19 and endocrine diseases. A statement from the European Society of Endocrinology. Endocrine 2020; 68: 2-5

[17] Rasmussen S, Thompson LA. Coronavirus disease 2019 and children. What Pediatric health care clinicians need to know. JAMA Pediatr 2020, doi: doi: 10.1001/jamapediatrics.2020.1224

[18] Guan W], Ni ZY, Hu Y et al. China Medical Treatment Expert Group for Covid-19. Clinical characteristics of coronavirus disease 2019 in China. N Engl J Med 2020; 382: 1708-1720

[19] COVID-19 Surveillance Group. Characteristics of COVID-19 patients dying in Italy: Report based on available data on March $20^{\text {th }}, 2020$. Rome, Italy: Instituto Superiore Di Sanita; 2020. Internet; Available from: https://www.epicentro.iss.it/ coronavirus/bollettino/Report-COVID-2019_20_marzo_eng.pdf

[20] CDC COVID-19 Response Team Preliminary Estimates of the Prevalence of Selected Underlying Health Conditions Among Patients with Coronavirus Disease 2019 - United States, February 12-March 28, 2020. MMWR Morb Mortal Wkly Rep 2020; 69: 382-386

[21] Guo W, Li M, Dong Y et al. Diabetes is a risk factor for the progression and prognosis of COVID-19. Diabetes Metab Res Rev 2020; e3319

[22] Kaiser UB, Mirmira RB, Stewart PM. Our response to COVID-19 as Endocrinologists and Diabetologists. J Clin Endocrinol Metab 2020; 105: 1-3

[23] Lazzerini M, Barbi E, Apicella A et al. Delayed Access or provision of care in Italy resulting from fear of COVID-19. Lancet Child Adolesc Health 2020; 4: e-10-e11

[24] Bancos I, Hazeldine J, Chortis V et al. Eur J Primary adrenal insufficiency is associated with impaired natural killer cell function: A potential link to increased mortality. Endocrinol 2017; 176: 471-480

[25] Baldeweg SE, Ball S, Brooke A et al. Society for Endocrinology clinical guidance: Inpatient management of cranial diabetes insipidus. Endocr Connect 2018; 7: G8-G11

[26] Tomlinson JW, Holden N, Hills RK et al. Association between premature mor- tality and hypopituitarism. West mid- lands prospective hypopituitary study group. Lancet 2001 ; 357: 425-431

[27] British Thyroid Foundation. Thyroid disease and coronavirus (COVID-19). Internet, cited 25 March 2020; Available from: https:// www.btf-thyroid.org/news/thyroid-disease-and-coronavirus-covid-19

[28] Senniappan S, Alexandrescu S, Tatevian N et al. Sirolimus therapy in infants with severe hyperinsulinemic hypoglycemia. N Engl J Med 2014; 370: 1131-1137
[29] Arlt W.Society for Endocrinology Clinical Committee Society for Endocrinology Endocrine Emergency Guidance: Emergency management of acute adrenal insufficiency (adrenal crisis) in adult patients. Endocr Connect 2016; 5: G1-G3

[30] Cushing's Disease News. Information about COVID-19 for Cushing's disease patients. Internet, cited 22 April 2020; Available from: https:// cushingsdiseasenews.com/information-about-covid-19-for-cushings-disease-patients/

[31] Qin ], Deng X, Wei A. Correlation between hypocalcemia and acute exacerbation of chronic obstructive pulmonary disease in the elderly. Postgrad Med 2019; 131: 319-323

[32] Andersen CA, Murphy KE, Fernandez ML. Impact of Obesity and Metabolic Syndrome on Immunity. Adv Nutr 2016; 7: 66-75

[33] Rundle AG, Park Y, Herbstman JB et al. COVID-19-Related School Closings and Risk of Weight Gain Among Children. Obesity 2020; 28: 1008-1009

[34] World Health Organization (WHO). Mental health and psychosocial considerations during the COVID-19 outbreak. Internet, cited 18 March 2020; Available from: https://www.who.int/docs/default-source/coronaviruse/mental-health-considerations.pdf

[35] CDC. Daily life and Coping. Internet, cited 16 April 2020; Available from: https://www.cdc.gov/coronavirus/2019-ncov/daily-life-coping/ managing-stress anxiety.html?CDC_AA_ refVal = https \%3A\%2F\%2Fwww.cdc.gov\%2Fcoronavirus \%2F2019ncov\%2Fprepare\%2Fmanaging-stress-anxiety.html

[36] Wei M, Yuan J, Liu Y et al. Novel coronavirus infection in hospitalized infants under 1 year of age in China. JAMA 2020; 323: 1313-1314

[37] Tan YP, Tan BY, Pan J et al. Epidemiologic and clinical characteristics of 10 children with coronavirus disease 2019 in Changsha, China. J Clin Virol 2020; 127: 104353

[38] Yuan R, Xu QH, Xia CC et al. Psychological status of parents of hospitalized children during the COVID-19 epidemic in China. Psychiatry Res 2020; 288: 112953

[39] Lee J. Mental health effects of school closures during COVID-19. Lancet Child Adolesc Health 2020, pii: S2352-4642(20)30109-7. doi: doi:10.1016/S2352-4642(20)30109-7 [Epub ahead of print]

[40] Public Health England. Guidance for parents and carers on supporting children and young people's mental health and wellbeing during the coronavirus (COVID-19) pandemic. Internet, cited 21 April 2020; Available from: https://www.gov.uk/government/publications/ covid-19-guidance-on-supporting-children-and-young-peoples-mental-health-and-wellbeing/guidance-for-parents-and-carers-on-supporting-children-and-young-peoples-mental-health-and-wellbeingduring-the-coronavirus-covid-19-outbreak

[41] Segerstrom SC, Miller GE. Psychological stress and the human immune system: A meta-analytic study of 30 years of inquiry. Psychol Bull 2004; 130: 601-630

[42] Cohen S, Doyle W], Skoner DP. Psychological stress, cytokine production, and severity of upper respiratory illness. Psychosom Med 1999; 61: 175-180

[43] Miller GE, Cohen S, Pressman S et al. Psychological stress and antibody response to influenza vaccination: when is the critical period for stress, and how does it get inside the body? Psychosom Med 2004; 66: 215-223

[44] Stam H, Hartman EE, Deurloo JA et al. Young adult patients with a history of pediatric disease: impact on course of life and transition into adulthood. J Adolesc Health 2006; 39: 4-13

[45] Nardi L, Zucchini S, D’Alberton F et al. Quality of life, psychological adjustment and metabolic control in youths with type 1 diabetes: a study with self and parent-report questionnaires. Pediatr Diabetes 2008; 9: 496-503

[46] Stawski M, Auerbach JG, Barasch M et al. Behavioural problems of children with chronic physical illness and their sibilings. Eur Child Adolescent Psychiatry 1997; 6: 20-25 